\title{
THE COEFFICIENTS OF QUASICONFORMALITY OF CONES IN $n$-SPACE
}

\author{
KARI HAG and M. K. VAMANAMURTHY
}

1. Introduction. In this paper we extend some results of Gehring and Väisälä [6] to $n$-space. The outer coefficient of a cylinder and that of a convex cone have been obtained by them in 3-space. We show that their methods can be modified to obtain outer coefficients of increasing convex as well as nonconvex cones and include cylinder as a limiting case of a convex cone. The problems of characterizing domains with finite coefficients and that of determining these coefficients are rather complicated in spaces of dimension greater than 2. Some results in this direction have been obtained in [1], [2], [3], and [6].

The authors wish to thank Professor F. W. Gehring for many helpful discussions.

2. Notation. We refer to [10] for all definitions and notations not explicitly stated here.

For each positive integer $p$, we let $\Omega_{p}$ denote the $p$-dimensional Lebesgue measure of $B^{p}$ and $\omega_{p-1}$ denote the $(p-1)$-dimensional Lebesgue measure of $S^{p-1}$. We observe that $\omega_{p-1}=p \Omega_{p}=2 \pi^{p / 2} / \Gamma_{p / 2}$, where $\Gamma$ is the classical Gamma function.

We let $\left(r, \theta, x_{n}\right)$ and $(t, \theta, \varphi)$ denote the cylindrical and spherical coordinates of $x=\sum_{i=1}^{n} x_{i} e_{i}$ in $R^{n}$. Here,

$$
\theta=\left(\theta_{1}, \theta_{2}, \ldots, \theta_{n-2}\right), \quad r \geqq 0, \quad t \geqq 0, \quad 0 \leqq \varphi, \quad \theta_{i} \leqq \pi,
$$

$1 \leqq i \leqq n-3$ and $0 \leqq \theta_{n-2}<2 \pi$. These coordinates are related by the formulas: $x_{n}=t \cos \varphi, r=t \sin \varphi, x_{1}=r \cos \theta_{1}, x_{2}=r \sin \theta_{1} \cos \theta_{2}, x_{3}=r \sin \theta_{1} \sin \theta_{2} \cos \theta_{3}, \ldots$, $x_{n-2}=r \sin \theta_{1} \sin \theta_{2} \ldots \cos \theta_{n-2}, x_{n-1}=r \sin \theta_{1} \sin \theta_{2} \ldots \sin \theta_{n-2}$. A domain $D$ in $\bar{R}^{n}$ is called a cone of angle $\alpha, 0<\alpha<\pi$, if $D$ can be mapped conformally onto

$$
C_{\alpha}=\{(t, \theta, \varphi): 0 \leqq \varphi<\alpha\},
$$

while $D$ is called a cylinder (cone of angle 0 ) if it can be conformally mapped 
onto

$$
C_{0}=\left\{\left(r, \theta, x_{n}\right): r<1\right\} .
$$

If $0 \leqq \alpha \leqq \pi / 2$, the cone is convex, while if $\pi / 2<\alpha<\pi$, it is nonconvex.

The inner, outer, and maximal coefficients of the ordered pair $\left(D, D^{\prime}\right)$ of domains in $\bar{R}^{n}$, are defined as

$$
\begin{aligned}
K_{I}\left(D, D^{\prime}\right) & =\inf K_{I}(f), \quad K_{O}\left(D, D^{\prime}\right)=\inf K_{O}(f), \\
K\left(D, D^{\prime}\right) & =\inf K(f),
\end{aligned}
$$

where the infima are taken over all homeomorphisms $f$ of $D$ onto $D^{\prime}$. It follows from (3) that

$$
\begin{gathered}
1 \leqq K_{I}\left(D, D^{\prime}\right), \quad K_{O}\left(D, D^{\prime}\right) \leqq \infty, \\
K_{I}\left(D, D^{\prime}\right)=K_{O}\left(D^{\prime}, D\right), \\
K_{I}\left(D, D^{\prime}\right) \leqq K_{O}^{n-1}\left(D, D^{\prime}\right),
\end{gathered}
$$

and that the coefficients are finite if and only if there exists a quasiconformal mapping from $D$ onto $D^{\prime}$. In this case we say that $D$ and $D^{\prime}$ are quasiconformally equivalent.

The following notation is used throughout the paper:

$$
\begin{gathered}
q(\varphi)=\int_{0}^{\varphi}(\sin u)^{\frac{2-n}{n-1}} d u, \quad 0 \leqq \varphi \leqq \pi, \\
0<\alpha<\beta<\pi, \text { given constants, } \\
c=q(\beta) / q(\alpha), \quad q\left(\varphi^{\prime}\right)=c q(\varphi), \text { for } 0 \leqq \varphi \leqq \alpha, \quad \text { and } \\
s(\varphi)=\frac{\sin \varphi^{\prime}}{\sin \varphi} \text { for } 0<\varphi \leqq \alpha, \quad s(0)=c^{n-1} .
\end{gathered}
$$

3. The results.

Lemma 1. Given $0<a<1$ and $b>-1$, let $f, g, h$ be functions on $[0, \pi]$ defined by

and

$$
\begin{gathered}
f(t)=\int_{0}^{t}(\sin u)^{b} d u, \\
g(t)=f^{-1}(a f(t)),
\end{gathered}
$$

$$
h(t)=\frac{\sin t}{\sin g(t)} \text { for } \quad t \neq 0
$$

$h(0)=a^{-1 /(b+1)}$. Then $h$ is continuous and decreasing.

Proof. Continuity follows from L'Hospital's rule applied to the $(b+1)$-th power of $h(t)$ as $t \rightarrow 0$. Next for monotonicity, by differentiating $h(t)$ and simplify- 
ing, we get

$$
h^{\prime}(t)=\frac{\cos t \cos g(t)}{(\sin g(t))^{b+2}} G(t)
$$

where $G(t)=(\sin g(t))^{b+1} \sec g(t)-a(\sin t)^{b+1} \sec t$, and $G^{\prime}(t)=a(\sin t)^{b}\left(\tan ^{2} g(t)-\right.$ $\left.-\tan ^{2} t\right)$. Now, on $(0, \pi / 2)$, since $g(t)<t$, it follows that $G^{\prime}, G$ and $h^{\prime}$ are all negative. Next, on $\left[\pi / 2, g^{-1}(\pi / 2)\right], h$ is clearly decreasing. Finally, on $\left(g^{-1}(\pi / 2), \pi\right), G^{\prime}$ is positive, whence $G$ and $h^{\prime}$ are negative.

Theorem 1. Given $0 \leqq \alpha<\beta<\pi$, let $D, D^{\prime}$ be cones of angles $\alpha$ and $\beta$, respectively. Then

$$
K_{O}\left(D, D^{\prime}\right) \leqq\left(\frac{q(\beta)}{q(\alpha)}\right)^{n-2}\left(\frac{\sin \alpha}{\sin \beta}\right)^{\frac{n-2}{n-1}},
$$

where for $\alpha=0$, the right side is replaced by its limit as $\alpha \rightarrow 0$, that is,

$$
q(\beta)^{n-2}\left((n-1)(\sin \beta)^{1 /(n-1)}\right)^{2-n} .
$$

Proof. We may assume that $D=C_{\alpha}$ and $D^{\prime}=C_{\beta}$ as in (1). First let $\alpha>0$. Let $f_{\alpha}^{\beta}=f: C_{\alpha} \rightarrow C_{\beta}$ be defined by

$$
\left(t^{\prime}, \theta, \varphi^{\prime}\right)=f(t, \theta, \varphi), \quad \text { where }
$$

$$
q\left(\varphi^{\prime}\right)=c q(\varphi), \quad \log t^{\prime}=c(s(\alpha))^{\frac{n-2}{n-1}} \log t
$$

$c, s$ as in (5). Then $f$ is a diffeomorphism whose stretchings at a point $(t, \theta, \varphi)$ are proportional to

$$
c(s(\alpha))^{\frac{n-2}{n-1}}, \quad c(s(\varphi))^{\frac{n-2}{n-1}} \text { and } \quad s(\varphi)
$$

where $s(\varphi)$ occurs $(n-2)$ times. From Lemma 1 it follows that the maximum of these stretchings is $c(s(\varphi))^{(n-2) /(n-1)}$, whence

$$
K_{O}(f)=c^{n-2}(s(\alpha))^{\frac{2-n}{n-1}},
$$

and (6) follows for $\alpha>0$.

Next for $\alpha=0$ we use a limiting argument as follows. For each $j \in N$, let $f_{\beta / j}^{\beta}=f_{j}: C_{\beta / j} \rightarrow C_{\beta}$ be defined as in (8). Let $S_{j}$ be the radial stretching of $R^{n}$ given by $S_{j}(x)=\cot (\beta / j) x$ and $T_{j}$ the translation $T_{j}(x)=x-\cot (\beta / j) e_{n}$. Then the sequence of mappings

$$
T_{j} \circ S_{j} \circ f_{j}^{-1}: C_{\beta} \rightarrow C_{\beta / j}-\cot (\beta / j) e_{n}
$$

converges $c$-uniformly ([10]) to a mapping $f^{-1}: C_{\beta} \rightarrow C_{0}$ and

$$
K_{o}(f)=K_{I}\left(f^{-1}\right) \leqq \varliminf_{j \rightarrow \infty} K_{o}\left(f_{j}\right)=q(\beta)^{n-2}\left((n-1)(\sin \beta)^{1 /(n-1)}\right)^{2-n},
$$

and (6) follows for $\alpha=0$. 
We next proceed to prove that there is indeed equality in (6) for $0 \leqq \alpha<\beta \leqq \pi / 2$ and for $\pi / 2 \leqq \alpha<\beta<\pi$. For this we need the generalizations of some modulus estimates in [6] for curve families in a cylinder and in a cone.

Lemma 2. Let $2 \leqq p \leqq n-1$ and let $\Gamma=\Gamma_{P}$ be the family of curves in $B^{p}(x, 1)$ joining its boundary $S^{p-1}(x, 1)$ to a given point $P \in B^{p}(x, 1)$ and let $\varrho \in F(\Gamma)$. Then

$$
\int_{R^{p}} \varrho^{p+1} d m_{p} \geqq p^{1-p} \Omega_{p}
$$

Proof. We may assume $P=0$. For each $y \in S^{p-1}$, let $\gamma_{y}$ be the segment joining 0 and $S^{p-1}(x, 1)$ through $y$. Then Hölder's inequaiity yields

$$
\begin{gathered}
1 \leqq\left(\int_{\gamma_{y}} \varrho d s\right)^{p+1} \leqq \int_{0}^{l\left(\gamma_{y}\right)} \varrho^{p+1} t^{p-1} d t\left(\int_{0}^{l\left(\gamma_{y}\right)} \frac{1-p}{t p} d t\right)^{p} \\
=l\left(\gamma_{y}\right) p^{p} \int_{0}^{l\left(\gamma_{y}\right)} \varrho^{p+1} t^{p-1} d t
\end{gathered}
$$

or

$$
\int_{0}^{l\left(\gamma_{y}\right)} \varrho^{p+1} t^{p-1} d t \geqq p^{-p} l\left(\gamma_{y}\right)^{-1} .
$$

Integrating with respect to $y$ we get

$$
\int_{R^{p}} \varrho^{p+1} d m_{p} \geqq p^{-1} \int_{S^{p-1}} l\left(\gamma_{y}\right)^{-1} d m_{p-1} .
$$

On the other hand, Hölder's inequality again yields

or

$$
\omega_{p-1}^{p+1}=\left(\int_{S^{p-1}} d m_{p-1}\right)^{p+1} \leqq \int_{S^{p-1}} l\left(\gamma_{y}\right)^{n-1} d m_{p-1}\left(\int_{S^{p}-1} l\left(\gamma_{y}\right)^{-1} d m_{p-1}\right)^{p},
$$

Thus

$$
\int_{S^{p-1}} l\left(\gamma_{y}\right)^{-1} d m_{p-1} \geqq p \Omega_{p}
$$

$$
\int_{R^{p}} \varrho^{p+1} d m_{p} \geqq p^{1-p} \Omega_{p} .
$$

Corollary 1. Given $0<a<b$, let $C$ be the finite part of the cylinder $C_{0}$, bounded by the planes $x_{n}=a$ and $x_{n}=b$, and let $E$ be a connected set in $C$ joining the bases of $C$. Let $\Gamma$ be the family of curves in $C$ joining $E$ to the lateral surface of $C$. Then

$$
M(\Gamma) \geqq \omega_{n-2}(b-a)(n-1)^{1-n},
$$

with equality if $E$ is the segment $\left\{t e_{n}: a<t<b\right\}$. 
Proof. Choose $\varrho \in F(\Gamma)$. For each $t \in(a, b)$, the plane $x_{n}=t$ has nonempty intersection with $E$ and meets $C$ in $B^{n-1}\left(t e_{n}, 1\right)$. Thus (10) with $p=n-1$ yields

$$
\int_{R^{n}} \varrho^{n} d m_{n} \geqq \int_{a}^{b} d t \int_{x_{n}=t} \varrho^{n} d m_{n-1} \geqq \omega_{n-2}(b-a)(n-1)^{1-n} .
$$

Next if $E$ is the segment $\left\{t e_{n}: a<t<b\right\}$, the function, $\varrho(x)=r^{(2-n) /(n-1)}(n-1)^{-1}$ for $x=\left(r, \theta, x_{n}\right) \in C$ and $\varrho(x)=0$ for $x \notin C$, is in $F(\Gamma)$ and

$$
\int_{R^{n}} \varrho^{n} d m_{n}=\omega_{n-2}(b-a)(n-1)^{1-n},
$$

thus there is equality in (11).

The proofs of the next lemma and its corollary are similar to those above and hence omitted ([6], [7]).

Lemma 3. Given $0<\alpha \leqq \pi / 2$, for $t>0$ let $T=C_{\alpha} \cap S^{n-1}(t)$ and $P \in T$. Let $\Gamma$ be the family of curves in $T$ joining $P$ and $\bar{T} \cap \partial C_{\alpha}$. Then $\varrho \in F(\Gamma)$ implies

$$
\int_{S^{n}-1(t)} \varrho^{n} d m_{n-1} \geqq \omega_{n-2} q(\alpha)^{1-n} t^{-1} .
$$

Corollary 2. Given $0<\alpha \leqq \pi / 2,0<a<b$, let $C$ be the part of $C_{\alpha}$ bounded by $S^{n-1}(a)$ and $S^{n-1}(b)$. Let $E$ be a connected set in $C$ joining the spherical bases of $C$ and let $\Gamma$ be the family of curves in $C$ joining $E$ to the lateral surface of $C$. Then

$$
M(\Gamma) \geqq \omega_{n-2} q(\alpha)^{1-n} \log \left(\frac{b}{a}\right),
$$

with equality if $E$ is the segment $\left\{t e_{n}: a<t<b\right\}$. Furthermore, the latter result holds for $0<\alpha<\pi$.

Lemma 4. Suppose that $f: \bar{C}_{0} \backslash\{\infty\} \rightarrow \bar{C}_{\pi / 2} \backslash\{0, \infty\}$ is a homeomorphism with

$$
\lim _{x_{n} \rightarrow-\infty} f(x)=0, \quad \lim _{x_{n} \rightarrow+\infty} f(x)=\infty,
$$

and that $f$ is $K$-quasiconformal in $C_{0}$. Then for each $a^{\prime}>0$, the set $T=$ $=f^{-1}\left(S^{n-1}\left(a^{\prime}\right) \cap \bar{C}_{\pi / 2}\right)$ lies between two planes $x_{n}=a_{1}$ and $x_{n}=a_{2}$ where

$$
0 \leqq a_{2}-a_{1} \leqq A K^{1 /(n-1)}, \quad A=A(n) .
$$

Proof. Let $a_{1}, a_{2}$ be the minimum and maximum of $x_{n}$, where $x \in T$. We may assume that $a_{1}<a_{2}$. Let $C$ be the finite part of $C_{0}$ bounded by the bases $x_{n}=a_{1}, x_{n}=a_{2}$, let $\Gamma$ be the family of curves in $C$ joining these bases and let $\Gamma^{\prime}=f(\Gamma)$. Then $([4],[10])$

$$
(1 / 2) H_{n}(1) \leqq M\left(\Gamma^{\prime}\right) \leqq K M(\Gamma)=K \Omega_{n-1}\left(a_{2}-a_{1}\right)^{1-n},
$$


where $H_{n}(r)$ is the modulus of the Teichmüller ring

$$
\bar{R}^{n} \backslash\left(C_{1} \cup C_{2}\right), \quad C_{1}=\left\{t e_{1}:-1 \leqq t \leqq 0\right\}, \quad C_{2}=\left\{t e_{1}: r \leqq t \leqq \infty\right\} .
$$

Thus (15) follows.

We next show that equality holds in (6) for increasing convex cones.

Theorem 2. Given $0 \leqq \alpha<\beta \leqq \pi / 2$, let $D, D^{\prime}$ be cones of angles $\alpha$ and $\beta$, respectively. Then

$$
K_{O}\left(D, D^{\prime}\right)=\left(\frac{q(\beta)}{q(\alpha)}\right)^{n-2} \cdot\left(\frac{\sin \beta}{\sin \alpha}\right)^{\frac{2-n}{n-1}} .
$$

Proof. Case (i): $\alpha=0, \beta=\pi / 2$. Let $f$ be any quasiconformal mapping of $C_{0}$ onto $C_{\pi / 2}$. Then $f$ can be extended to a homeomorphism of $\bar{C}_{0} \backslash\{\infty\}$ onto $\bar{C}_{\pi / 2} \backslash\{0, \infty\}$ ([10]). Further, by composing with a Möbius transformation, we may assume that

$$
\lim _{x_{n} \rightarrow-\infty} f(x)=0 \text { and } \lim _{x_{n} \rightarrow+\infty} f(x)=\infty .
$$

Now choose $0<a^{\prime}<b^{\prime}$ and set $C^{\prime}=\left(B^{n}\left(b^{\prime}\right) \backslash \bar{B}^{n}\left(a^{\prime}\right)\right) \cap C_{\pi / 2}$,

$$
E^{\prime}=\left\{t e_{n}: a^{\prime}<t<b^{\prime}\right\}, \quad T^{\prime}=B^{n-1}\left(b^{\prime}\right) \backslash \bar{B}^{n-1}\left(a^{\prime}\right), \quad S^{\prime}=R^{n-1} .
$$

Next let $\Gamma_{1}^{\prime}=\Delta\left(E^{\prime}, S^{\prime} ; C^{\prime}\right), \Gamma_{2}^{\prime}=\Delta\left(S^{n-2}\left(a^{\prime}\right), S^{n-2}\left(b^{\prime}\right) ; T^{\prime}\right)$. Then (15) implies that $f^{-1}$ maps $S^{n-1}\left(a^{\prime}\right) \cap C_{\pi / 2}$ and $S^{n-1}\left(b^{\prime}\right) \cap C_{\pi / 2}$ into $a_{1} \leqq x_{n} \leqq a_{2}$ and $b_{1} \leqq x_{n} \leqq b_{2}$, respectively, where

$$
0 \leqq a_{2}-a_{1}, \quad b_{2}-b_{1} \leqq A K(f)^{1 /(n-2)} .
$$

By choosing $a^{\prime}$ small enough we may also assume that $a_{2}<b_{1}$. Then (11) yields

$$
\frac{\left(b_{1}-a_{2}\right) \omega_{n-2}}{(n-1)^{n-1}} \leqq M\left(\Gamma_{1}\right) \leqq K_{O}(f) M\left(\Gamma_{1}^{\prime}\right)=K_{O}(f) \frac{\omega_{n-2}}{q(\pi / 2)^{n-1}} \log \left(b^{\prime} / a^{\prime}\right),
$$

and by the boundary correspondence property of $f$ ([5], [7]) we get Thus

$$
\frac{\omega_{n-2}}{\left(b_{2}-a_{1}\right)^{n-2}} \leqq M^{S}\left(\Gamma_{2}\right) \leqq K_{O}(f) M^{S^{\prime}}\left(\Gamma_{2}^{\prime}\right)=K_{O}(f) \omega_{n-2}\left(\log \left(b^{\prime} / a^{\prime}\right)\right)^{2-n} .
$$

$$
K_{O}(f) \geqq\left(\frac{q(\pi / 2)}{n-1}\right)^{n-2}\left(\frac{b_{1}-a_{2}}{b_{2}-a_{1}}\right)^{\frac{n-2}{n-1}} .
$$

Now letting $a^{\prime} \rightarrow 0$ and $b^{\prime} \rightarrow \infty$ and using (17) it follows that

$$
K_{o}(f) \geqq\left(\frac{q(\pi / 2)}{n-1}\right)^{n-2} \text {. }
$$

Combining this with (6), the result follows.

Case (ii): $0 \leqq \alpha<\beta \leqq \pi / 2$. Let $f$ be any quasiconformal mapping of $D=C_{\alpha}$ onto $D^{\prime}=C_{\beta}$. Let $f_{0}^{\alpha}: C_{0} \rightarrow C_{\alpha}$ and $f_{\beta}^{\pi / 2}: C_{\beta} \rightarrow C_{\pi / 2}$ be the mappings as in Theorem 
1. Then $g=f_{\beta}^{\pi / 2} \circ f \circ f_{0}^{\alpha}$ is a quasiconformal mapping of $C_{0}$ onto $C_{\pi / 2}$ and from (9) and (18) it follows that

$$
K_{O}(f) \geqq\left(\frac{q(\beta)}{q(\alpha)}\right)^{n-2}\left(\frac{\sin \alpha}{\sin \beta}\right)^{\frac{n-2}{n-1}},
$$

which together with (6) yields (16).

The next two lemmas will be needed in extending (16) for increasing nonconvex cones. We omit their proofs since they are similar to those of the previous lemmas (see [6], [7]).

Lemma 5. Given $0<\beta<\pi, 0<a<b$, let $\Gamma$ be the family of curves in $S=\partial C_{\beta} \cap B^{n}(b) \backslash \bar{B}^{n}(a)$ joining its boundary spheres. Then

$$
M^{S}(\Gamma)=\omega_{n-2}\left(\frac{\sin \beta}{\log (b / a)}\right)^{n-2} .
$$

Lemma 6. Given $0<\beta<\pi$, let $f: \bar{C}_{\pi / 2} \rightarrow \bar{C}_{\beta}$ be a homeomorphism, $f(0)=0$, $f(\infty)=\infty$ and let $f$ be K-quasiconformal in $C_{\pi / 2}$. Then for each $a^{\prime}>0$, the set $f^{-1}\left(S^{n-1}\left(a^{\prime}\right) \cap \bar{C}_{\beta}\right)$ lies in $\bar{B}^{n}\left(a_{2}\right) \backslash B^{n}\left(a_{1}\right)$, where

$$
1 \leqq a_{2} / a_{1} \leqq A, \quad A=A(n, \beta, K) .
$$

Theorem 3. Given $\pi / 2 \leqq a<\beta<\pi$, let $D, D^{\prime}$ be cones of angles $\alpha, \beta$, respectively. Then

$$
K_{O}\left(D, D^{\prime}\right)=\left(\frac{q(\beta)}{q(\alpha)}\right)^{n-2}\left(\frac{\sin \alpha}{\sin \beta}\right)^{\frac{n-2}{n-1}} .
$$

Proof. As in the proof of Theorem 2 we consider two cases.

Case (i): Let $\alpha=\pi / 2$. We may assume that $D=C_{\pi / 2}, D^{\prime}=C_{\beta}$. Let $f$ be any quasiconformal mapping of $D$ onto $D^{\prime}$. Then $f$ can be extended to a homeomorphism of $\bar{D}$ onto $\bar{D}^{\prime}$ ([10]) with $f(0)=0, f(\infty)=\infty$. Now choose $0<a^{\prime}<b^{\prime}$ and set $C^{\prime}=D^{\prime} \cap B^{n}\left(b^{\prime}\right) \backslash \bar{B}^{n}\left(a^{\prime}\right), \quad E^{\prime}=\left\{t e_{n}: a^{\prime}<t<b^{\prime}\right\}, \quad T^{\prime}=\partial D^{\prime} \cap B^{n}\left(b^{\prime}\right) \backslash \bar{B}^{n}\left(a^{\prime}\right)$, $S^{\prime}=\partial D^{\prime}, \quad \Gamma_{1}^{\prime}=\Delta\left(E^{\prime}, S^{\prime} ; C^{\prime}\right), \quad \Gamma_{2}^{\prime}=\Delta\left(S^{n-1}\left(a^{\prime}\right), \quad S^{n-1}\left(b^{\prime}\right) ; T^{\prime}\right)$. Next $f^{-1}$ maps $\bar{D}^{\prime} \cap S^{n-1}\left(a^{\prime}\right)$ and $\bar{D}^{\prime} \cap S^{n-1}\left(b^{\prime}\right)$ into $\bar{B}^{n}\left(a_{2}\right) \backslash B^{n}\left(a_{1}\right)$ and $\bar{B}^{n}\left(b_{2}\right) \backslash B^{n}\left(b_{1}\right)$, respectively, where

$$
1 \leqq a_{2} / a_{1}, \quad b_{2} / b_{1} \leqq A .
$$

By choosing $a^{\prime}$ small enough, we may assume that $a_{2}<b_{1}$. Then Corollary 2 yields

$$
\frac{\omega_{n-2}}{q(\pi / 2)^{n-2}} \log \left(\frac{b_{1}}{a_{2}}\right) \leqq M\left(\Gamma_{1}\right) \leqq K_{o}(f) M\left(\Gamma_{1}^{\prime}\right)=K_{o}(f) \frac{\omega_{n-2}}{q(\beta)^{n-1}} \log \left(\frac{b^{\prime}}{a^{\prime}}\right),
$$

and by the boundary correspondence property of $f$ ([5], [7]) we get

$$
\frac{\omega_{n-2}}{\left(\log \left(b_{2} / a_{1}\right)\right)^{n-2}} \leqq M^{S}\left(\Gamma_{2}\right) \leqq K_{o}(f) M^{S^{\prime}}\left(\Gamma_{1}^{\prime}\right)=K_{o}(f) \omega_{n-2}\left(\frac{\sin \beta}{\log \left(b^{\prime} / a^{\prime}\right)}\right)^{n-2}
$$


Thus combining the above two inequalities, letting $a^{\prime} \rightarrow 0$ and using (21), we get

$$
K_{O}(f) \geqq\left(\frac{q(\beta)}{q(\pi / 2)}\right)^{n-2}(\sin \beta)^{\frac{2-n}{n-1}} .
$$

Hence (6) and (22) yield (20) for the case $\alpha=\pi / 2$.

Case (ii): Let $\pi / 2<\alpha<\beta<\pi$. As before we may assume that $D=C_{\alpha}, D^{\prime}=C_{\beta}$. Let $f$ be any quasiconformal mapping of $D$ onto $D^{\prime}$ and let $f_{\pi / 2}^{\alpha}: C_{\pi / 2} \rightarrow C_{\alpha}$, be the mapping as in Theorem 1. Then $g=f_{\pi / 2}^{\alpha} \circ f$ is a quasiconformal mapping of $C_{\pi / 2}$ onto $C_{\beta}$, whence from (9) and (22), it follows that

$$
K_{o}(f) \geqq\left(\frac{q(\beta)}{q(\alpha)}\right)^{n-2}\left(\frac{\sin \alpha}{\sin \beta}\right)^{\frac{n-2}{n-1}},
$$

which together with (6) yields (20).

Remark. If $0 \leqq \alpha<\beta \leqq \pi / 2$ or $\pi / 2 \leqq \alpha<\beta<\pi$, then (9), (16) and (20), imply that the mapping $f_{\alpha}^{\beta}: C_{\alpha} \rightarrow C_{\beta}$, is extremal for the outer coefficient $K_{O}\left(C_{\alpha}, C_{\beta}\right)$. For $\alpha<\pi / 2<\beta$, the problem is still open.

Given a domain $D$ in $\bar{R}^{n}$, a point $P \in \partial D$ is said to be a cone point for $D$ of angle $\alpha, 0<\alpha<\pi$, if there exists a neighborhood $V$ of $P$ and a cone $G$ of vertex $P$, angle $\alpha$, such that $V \cap D=V \cap G$. Theorems 2 and 3 together with the fact that a cone is ray like at its vertex yield sharp lower bounds for outer dilatation of mappings of a class of domains. This result is analogous to Theorem 9 in [6] and Theorem 40.3 in [10].

Theorem 4. Let $D, D^{\prime}$ be domains in $\bar{R}^{n}$ which have cone points $P, Q$ of angles $\alpha, \beta$, respectively, where $0<\alpha<\beta<\pi$. Let $f$ be a homeomorphism of $D$ onto $D^{\prime}$ such that $Q$ is a cluster point of $f$ at $P$. Then

$$
K_{O}(f) \geqq\left(\frac{q(\beta)}{q(\alpha)}\right)^{n-2}\left(\frac{\sin \alpha}{\sin \beta}\right)^{\frac{n-2}{n-1}},
$$

and the bound is sharp.

In the above discussion we have only considered the outer coefficient for increasing cones. In view of (4) we get analogous results for the inner coefficient for decreasing cones. However, the problem of determining the inner coefficients for increasing cones is still open. Of course, rough upper and lower bounds for this case can be obtained by obvious $n$-dimensional analogues of Theorem 9.2 in [6] and Theorem 3.2 in [11]. 


\section{References}

[1] Gauld, David B., and M. K. Vamanamurthy: Quasiconformal extensions of mappings in $n$-space. - Ann. Acad. Sci. Fenn. Ser. A I 3, 1977, 229-246.

[2] Gehring, F. W.: Extension of quasiconformal mappings in three space. - J. Analyse Math. 14, $1965,171-182$.

[3] Gehring, F. W.: Extension theorems for quasiconformal mappings in $n$-space. - Ibid. 19, 1967, 149-169.

[4] Gehring, F. W.: Lectures on quasiconformal mappings. - Unpublished, Institut Mittag-Leffler, 1972.

[5] Gehring, F. W.: Dilatations of quasiconformal boundary correspondences. - Duke Math. J. 39, 1972. 89-95.

[6] Gehring, F. W., and J. VÄISÄL ̈̈: The coefficients of quasiconformality of domains in space. - Acta. Math. 114, 1965, 1-70.

[7] HAG, KARI: Quasiconformal boundary correspondence and extremal mappings. - Thesis, University of Michigan, Ann Arbor, Michigan, USA, 1972.

[8] Väıs̈̈LÄ, J.: On quasiconformal mappings in space. - Ann. Acad. Sci. Fenn. Ser. A I 298, 1961, $1-36$.

[9] V̈̈ISÄLÄ, J.: On quasiconformal mappings of a ball. - Ibid. 304, 1961, 1-7.

[10] VÄISÄLÄ, J.: Lectures on $n$-dimensional quasiconformal mappings. - Lecture Notes in Mathematics 229, Springer-Verlag, Berlin-Heidelberg-New York, 1971.

[11] Vamanamurthy, M. K.: Quasiconformal mappings in space. - Thesis, University of Michigan, Ann Arbor, Michigan, USA, 1969.

University of Trondheim

Department of Mathematics, NTH

N-7034 Trondheim

Norway

Received 17 August 1977
University of Michigan

Department of Mathematics

Ann Arbor, Michigan 48109

USA

University of Auckland

Department of Mathematics

Auckland

New Zealand 